\title{
FORMATION OF BRAGG GRATINGS IN GERMANIUM DOPED OPTICAL FIBERS USING A PRISM INTERFEROMETER
}

\author{
M. DOUAY, S. CANON, S. LEGOUBIN, E. FERTEIN, P. BERNAGE, P. NIAY, \\ J.F. BAYON* and T. GEORGES* \\ Laboratoire de Spectroscopie Moléculaire, Unité de Recherche Associée au C.N.R.S. $n^{\circ} 779$, \\ Université de Lille Flandres Artois, U.F.R. de Physique, Bâtiment P5, \\ F-59655 Villeneuve d'Ascq, France \\ ${ }^{*}$ C.N.E.T. Lannion, route de Trégastel, BP. 40, F-22301 Lannion, France
}

\begin{abstract}
Bragg gratings have been written in Germanium doped core silica fibers by transversally exposing the fibers to fringe patterns produced by a pair of coherent U.V. pulsed beams combined inside a prism interferometer. The transmission spectra of the gratings have been recorded at various times of irradiation. The drop in transmission near resonance wavelength may be as large as 100\%, depending on the experimental conditions of the writing experiment. The thermal annealing behavior of different gratings shows that the evolution of the fiber transmittivity recovery versus temperature depends upon the number and the energy of the laser pulses applied to form the grating and upon the nature of the fiber.
\end{abstract}

\section{Introduction}

Grating assited fiber devices are of increasing importance in numerous applications including, for instance, optical communications, signal processing and all fiber laser or distributed fiber sensors. Thus, a new class of such optical fiber devices that make use of a permanently photoinduced index grating within the core of $\mathrm{GeO}_{2}$ doped fibers has been developed recently $/ 1 / / 2 / / 3 /$. In these devices, a longitudinal periodic change of the refractive index is produced by a spatially periodic transversal laser illumination. Under resonance conditions, the periodic perturbation leads to a power coupling between the counterpropagating fundamental modes through a monomode fiber $/ 1 / / 3 /$ (Bragg reflector), or between the forward $L_{0} P_{1}$ and $L P_{1}$ modes /2/ through a bimodal fiber (mode convertor). The wavelength of the U.V. laser radiation is selected to lie in the oxygen-vacancy defect band of germania near $245 \mathrm{~nm}$. The exact mechanisms that form the gratings are not yet fully understood, but are probably related to the creation of $\mathrm{Ge}(\mathrm{I}), \mathrm{Ge}$ (II) or Ge(III) defect centers via the electrons removed from the bleaching of the $245 \mathrm{~nm}$ absorption band /4/ /5/. The thermal annealing behavior of these paramagnetic defect center E.P.R. spectra has been studied in $X$ irradiated Ge doped silica /6/ and in broadband U.V. irradiated Ge fibers /7/. From these experiments, it appears that the relative concentrations of $\mathrm{Ge}$ (I), $\mathrm{Ge}$ (II) or Ge(III) defects within the sample are quite dependent on its temperature, with the Ge(III) defect being the most stable center to heat. Therefore, it is important from both technological and fundamental points of view to caracterize the growth of a Bragg filter written in a monomode fiber 
versus irradiation time and to follow its transmittivity recovery in the course of filter thermal annealing experiments.

\section{Experimental}

The method for producing the gratings uses a modified form of the interferometer described by $S$. Chandra et al $/ 8 /$. The output beam from an Xecl pumped dye laser is frequency doubled through a B.B.O. crystal. The pulsed U.V. radiation $\left(E_{p} \simeq 1.5 \mathrm{~mJ}\right.$ per pulse, $20 \mathrm{~Hz}$ maximum frequency rate, $20 \mathrm{~ns}$ pulse length, $0.4 \mathrm{~cm}^{-1}$ spectral width) is tuned near $243 \mathrm{~nm}$. This $243 \mathrm{~nm}$ beam, expanded by an afocal telescope $(x$ 16), impinges on the hypothenuse face of an isosceles right-angle fused silica prism. The prism works as an interferometer, splitting the U.V. beam into two equal intensity halves which are combined to interfere on one face of the prism. A fiber is placed in contact with the prism surface, normal to the fringe direction. The intensity of the interference pattern is increased by focusing the U.V. beam on the fiber using a cylindrical lens. The spot dimensions near the axis of the fiber are typically $3 \mathrm{~mm} \times 100 \mu \mathrm{m}$. By rotating the prism assembly, the angle of incidence of the $243 \mathrm{~nm}$ beam can be varied to allow the tuning of the grating period and consequently of the Bragg resonance wavelength $\lambda_{B}$. A white light source is used with a high resolution spectrometer $\left(R_{0}=240000\right)$ to record the transmission spectrum of the fiber near $\lambda_{B}$ after a burst of $U . V$. pulses has irradiated the fiber to write the grating. The minimum of the filter transmittivity and the FWHM bandwidth can then be measured for various shot numbers. To follow the filter transmittivity recovery induced through a thermal annealing experiment, several gratings are written on a fiber at several wavelengths $\lambda_{i}\left(1.18 \mu \mathrm{m}<\lambda_{i}<1.19 \mu \mathrm{m}\right)$ under different experimental conditions. Thus, at room temperature, the transmittivity (or the bandwidth) is different for each grating. We increase step by step the temperature of the fiber placed in an oven. After 30 minutes at a fixed temperature, the transmission spectrum of the fiber is recorded within the range $1.18 \mu \mathrm{m}$ and $1.19 \mu \mathrm{m}$.

\section{Results}

Most of the results presented here have been obtained from a fiber manufactured by the Lannion-C.N.E.T. Laboratory (core diameter $5 \mu \mathrm{m}$, $\lambda_{c} \simeq 1.15 \mu \mathrm{m}, 11.5$ mole per cent $\mathrm{GeO}_{2}$ core doping, $B$ and $P$ cladding doping). Figure 1 shows the reflectivity growths of three Bragg gratings written at $\lambda_{B}=1.19 \mu \mathrm{m}$ versus shot number (solid lines). The parameter in each plot is the energy per pulse impinging on the prism. The reflectivity $R$ is calculated under the assumption that there is no loss at resonance and consequently that the relation $R=1-T$ is valid. Using the results of reference $/ 9 /$, we estimate the strength of the index perturbation when $R=0.9$ to be $\Delta n / n \simeq 2.6 \times 10^{-4}$. This value is probably underestimated because the intensity and the visibility of the interference pattern decrease when the interference order increases. These variations are not taken into account in the model used in reference $/ 9 /$. The dotted line of figure 1 shows the evolution of the spectral bandwidth of the $c$ filter versus shot number.

The thermal annealing behavior of four gratings is displayed on figure 2. The initial reflectivity of the $G_{1}$ grating (written by 5000 shots, $0.55 \mathrm{~mJ}$ incident on the prism) was saturated, its linewidth being $1 \mathrm{~nm}$ at room temperature. The $G_{2}$ and $G_{4}$ gratings were written, respectively, by 3000 and 1000 shots ( $0.55 \mathrm{~mJ}$ per impulsion) and the $G_{3}$ grating by 1000 shots $\left(0.65 \mathrm{~mJ}\right.$ per impulsion). At $100^{\circ} \mathrm{C}$, the reflec- 
tivities and/or the linewidths of the gratings begin to decrease. This result is in disagreement with the observations of G. Meltz et al /1/, who did not observe any change in the reflectivity or line-shape of a grating written in a commercial fiber which was heated to $500^{\circ} \mathrm{C}$ and maintained at that temperature for $18 \mathrm{~h}$. Another thermal annealing experiment performed in our laboratory using a different fiber $(2.5 \mu \mathrm{m}$ core diameter, $\lambda_{c}=1.12 \mu \mathrm{m}, 28$ mole per cent $\mathrm{GeO}_{2}$ core doping, $\mathrm{F}$ and $\mathrm{P}$ cladding doping) led to a good agreement with the observation of $G$. Meltz et al, provided that the illumination time applied to the fiber to obtain the grating was long enough (the grating was obtained from 32000 shots, $E_{\mathrm{p}}=0.55 \mathrm{~mJ}$, spot dimensons $\simeq 4 \mathrm{~mm} \times 500 \mu \mathrm{m}$, reflectivity at room temperature $\mathrm{R}=0.7$ ).

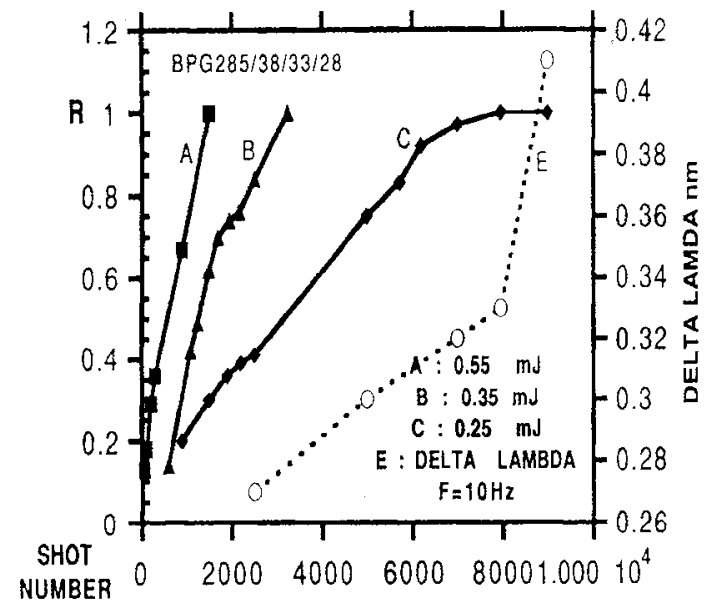

Fig. 1.- Reflectivity and spectral bandwidth variation versus shot number

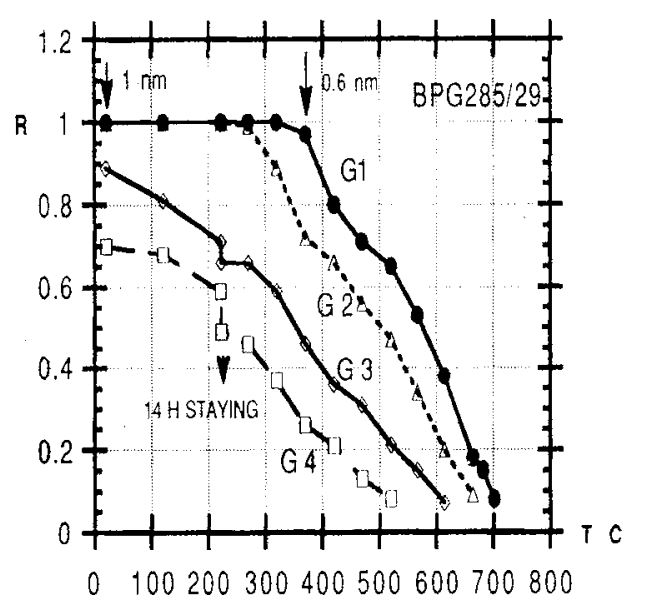

Fig. 2.- Thermal annealing behavior of four Bragg gratings written for different irradiation times on the fiber

\section{Discussion}

From the observations of references $/ 6 /$ and $/ 7 /$, it appears that the Ge(II) centers begin to anneal at room temperature. They are almost depleted at an annealing temperature of $350^{\circ} \mathrm{C}$. On the other hand, the $\mathrm{Ge}\left(\right.$ III) centers are stable up to $\simeq 500^{\circ} \mathrm{C}$. Assuming that the index variation leading to a grating formation results from the creation of $\mathrm{Ge}$ (II) and $\mathrm{Ge}$ (III) centers, we deduce that the relative concentration of these defects depends strongly upon the irradiation conditions and upon the fiber characteristics.

This work is developed from a C.N.E.T. contract $n^{\circ} 908$ B 076 . 


\section{References}

/1/ G. Meltz, w.พ. Morey and w.H. Glenn, Optics Letters $14, \mathrm{n}^{\circ} 15$, p. 823 (August 1, 1989).

/2/ K.O. Hill, B. Malo, K.A. Vineberg, F. Bilodeau, D.C. Johnson and $I$. Skinner, Electronic Letters $26, \mathrm{n}^{\circ} 16, \mathrm{p} .1270$ (August, 1990).

/3/ R. Kashyap, J.R. Armitage, R. Wyatt, S.T. Davey and D.I. Williams, Electronic Letters 26, $\mathrm{n}^{\circ} 11$, p. 730 (May, 1990).

/4/ D.P. Hand and P. St. J. Russel, Opt. Letters 15, n 2, p. 102 (January, 1990).

/5/ J.P. Bernardin and N.M. Lawandy, Opt. Comm. 79, ns 3-4, p. 194 (October, 1990).

/6/ E.J. Friébele and D.L. Griscom, Mat. Res. Soc. Symp. Proceedings, p. 319 (1986).

/7/ J. Simpson, J. Ritger and Di Marcello, Mat. Res. Soc. Symp. Proceedings, p. 333 (1986).

/8/ S. Chandra, w. Takeuchi and S.R. Hartman, Appl. Phys. Lett. 21, $n^{\circ} 4$, p. 144 (1972).

19/ D.K.W. Lam and B.K. Garside, Appl. Opt. 20, p. 440 (1981). 